\title{
A theory of metamaterials based on periodically loaded transmission lines: Interaction between magnetoinductive and electromagnetic waves
}

\author{
R. R. A. Syms ${ }^{\text {a) }}$ \\ Optical and Semiconductor Devices Group, Electrical and Electronic Engineering (EEE) Department, \\ Imperial College, Exhibition Road, London SW7 2BT, United Kingdom \\ E. Shamonina \\ Department of Physics, University of Osnabruck, 49060 Osnabruck, Germany \\ V. Kalinin \\ Transense Technologies Ltd. Upper Heyford, Bicester, Oxon OX25 5HD, United Kingdom \\ L. Solymar \\ Optical and Semiconductor Devices Group, Electrical and Electronic Engineering (EEE) Department, \\ Imperial College, Exhibition Road, London SW7 2BT, United Kingdom
}

(Received 10 June 2004; accepted 22 November 2004; published online 11 March 2005)

\begin{abstract}
The propagation of waves in a metamaterial consisting of split ring resonators (SRRs) and metallic rods is considered in several steps. The first involves the rods in isolation, the second the SRRs in isolation, and the third a combination of the two, which includes the coupling between neighboring SRRs and allows the propagation of magnetoinductive (MI) waves. The mathematical formulation is based on a conventional description of loaded transmission lines. A dispersion equation is derived to show the main features of known experimental results, including all the stop bands and passbands, the latter exhibiting both forward and backward waves. The interaction between electromagnetic and MI waves is presented in the form of a coupled dispersion equation. The applicability of the approaches based on negative material parameters is discussed. (C) 2005 American Institute of Physics. [DOI: 10.1063/1.1850182]
\end{abstract}

\section{INTRODUCTION}

The experimental results of Smith $e t$ al. ${ }^{1,2}$ and Shelby et $a l .{ }^{3,4}$ with split ring resonators (SRRs), ${ }^{5}$ metallic rods, ${ }^{6}$ and their combinations displayed some striking phenomena, which were fully corroborated by later measurements. ${ }^{7,8}$ The main aim was to confirm Veselago's prediction ${ }^{9}$ that negative refraction may occur when the effective permeability and the effective permittivity of a material are both negative. This correlation between negative material parameters and negative refraction has now been widely accepted.

It is certainly a possible explanation, but is it the only explanation? It seems rather artificial to have to invoke negative material parameters when the physics consists of nothing more than coupling of waves to a periodic array. In fact, an alternative explanation has been proposed by Lindell et $a l .{ }^{10}$ based on the familiarity of microwave engineers with backward wave amplifiers, oscillators, and antennas. ${ }^{11,12}$ The authors maintained that negative refraction is simply a consequence of the existence of backward waves (BW) and they even proposed that all the media having negative parameters should be referred to as BW media instead.

The present paper addresses the questions on whether we could still explain the experimental results quoted above if the concept of negative material parameters had never been proposed, and on what kind of mathematical apparatus that would require. The principal effects to explain are

${ }^{a)}$ Electronic mail: r.syms@imperial.ac.uk (i) the exponential decay of the input electromagnetic wave below a certain frequency when only a set of metallic rods is present;

(ii) the exponential decay of the input electromagnetic wave in a certain frequency band when only a periodic array of SRRs is present;

(iii) transmission with moderate attenuation when the frequency is within the stop band of the SRRs and below the cut-off frequency of the rods; and

(iv) refraction according to an inverse Snell's law when a wave is incident upon an ordinary (right handed in Veselago's terminology) medium from one satisfying condition (iii).

We assert here that all these phenomena can be explained by using nothing more sophisticated than the standard methods of loaded transmission line theory known to engineers at least for half a century. These were established by Brillouin ${ }^{13}$ and have been widely used to describe the properties of artificial dielectrics (see, for example, the book by Collin $^{14}$ ).

Apart from the main body of the experiments concentrating on the negative material parameter aspects of metamaterials, there is another set of experimental results available concerned with waves on an array of magnetically coupled resonant elements, namely, capacitively loaded loops, ${ }^{15}$ 'Swiss Rolls', ${ }^{16}$ and SRRs. ${ }^{17}$ It should be emphasized that these waves, known as magnetoinductive (MI) waves, are not simple analogs of longitudinal plasma waves. A longitudinal plasma wave (which has its electric-field vector in the longitudinal direction) exists only at a single frequency and 


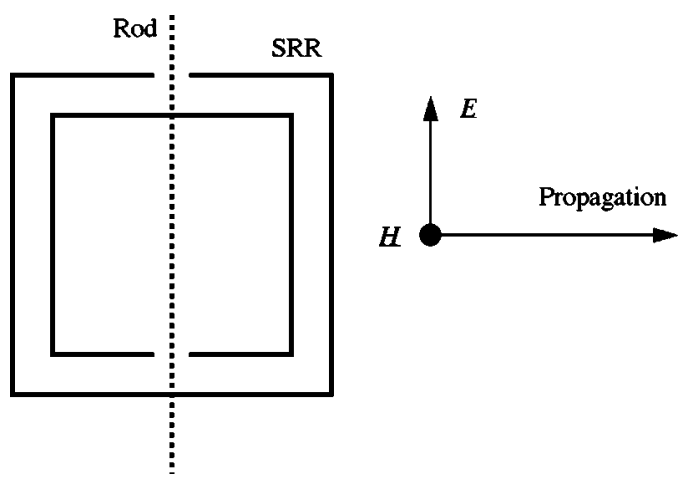

FIG. 1. Schematic of an electromagnetic wave incident upon an element of an array composed of split ring resonators and rods.

will not couple to a transverse electromagnetic wave. MI waves exist within a band of frequencies and do couple to electromagnetic waves. The transverse magnetic field of an input electromagnetic wave will, via the currents set up in the resonant elements, influence not only the electromagnetic wave itself but will also set up the MI waves.

These concepts have been discussed in several theoretical publications ${ }^{18,19}$ that do not invoke negative material parameters, and indeed it would not be easy to fit the MI waves into negative parameter theories. If one accepts that the effective permeability may be negative then it would be difficult to argue that the relative permeability seen by the MI wave is only that of free space. We do not wish here to prejudge the issue; a theory might be developed that includes both negative material parameters and MI waves. However, there is no other theory at the moment that could explain all the four phenomena above and could, in addition, incorporate $\mathrm{MI}$ waves.

The theory we present here is a one-dimensional one. It uses only circuit elements to model the well-known array of SRRs and rods, a unit of which is shown in Fig. 1. It needs to be emphasized that in our circuit approach there are no free parameters. Each one of the circuit elements is related to the actual properties of the array. We do, however, make the simplifying assumption that losses may be neglected. In Secs. II and III we consider the loading of a transmission line by an inductance and by a resonant circuit, respectively, to simulate the effect of metallic rods and SRRs. In Sec. IV a combined loading is considered which makes allowance for the propagation of MI waves as well. There is a discussion in Sec. V concerned with the role of negative material parameters and whether they are necessary. Conclusions are drawn in Sec. VI.

\section{PERIODIC LOADING OF A TRANSMISSION LINE BY METALLIC RODS}

A short metallic rod of length $a$ and radius $r$ can be characterized by a self-inductance $L_{r}$. Its value can be found in text books published some time ago (see, e.g., Ref. 20). The inductance of such a rod in a cubic lattice of side $a$ may be found as

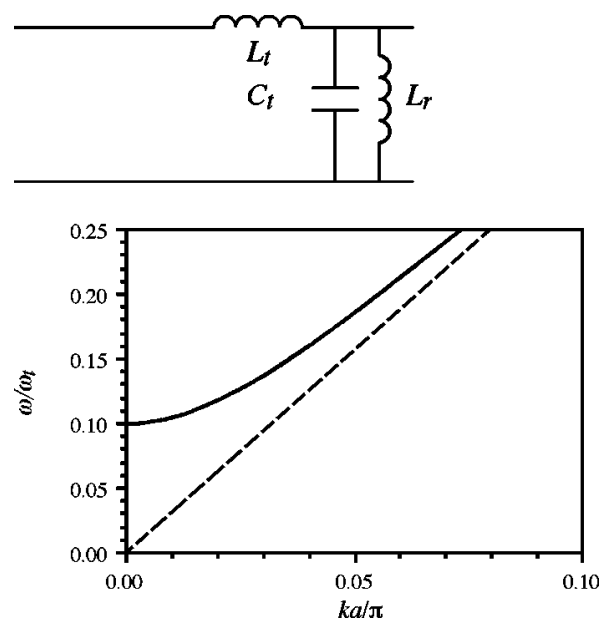

FIG. 2. (a) Equivalent circuit of length $a$ of a transmission line loaded with metal rods; (b) dispersion diagram predicted by Eq. (2) with $\omega_{p} / \omega_{t}=0$ (dotted line) and $\omega_{p} / \omega_{t}=0.1$ (full line).

$$
L_{r}=\left(\mu_{o} a / 2 \pi\right)[\ln (2 a / r)-1] .
$$

Here $\mu_{o}$ is the permeability of free space. A section of a transmission line of length $a$ loaded by such an inductance is shown in Fig. 2(a) in the form of a four pole. The corresponding dispersion equation for a periodic array can be readily obtained from Brillouin's theory, ${ }^{13}$ which is at the same time astonishingly simple and amazingly powerful. Once the loading impedance is inserted in the transmission line the problem is reduced to finding the transfer-matrix coefficients of the corresponding four pole and then the dispersion equation is obtained by equating the sum of the main diagonal elements with $2 \cos (k a)$. A little arithmetical effort will lead to

$$
4 \sin ^{2}(k a / 2)=\left(\omega^{2}-\omega_{p}^{2}\right) / \omega_{t}^{2} .
$$

Here $\omega$ is the angular frequency, $k a$ is the phase change per period, $\omega_{p}^{2}=\left(L_{r} C_{t}\right)^{-1}, \omega_{t}^{2}=\left(L_{t} C_{t}\right)^{-1}$, and $L_{t}$ and $C_{t}$ are the inductance and capacitance per length $a$ of the transmission line. If the medium is free space then $L_{t}=\mu_{o} a, C_{t}=\varepsilon_{o} a$, and $\omega_{t}=c / a$ where $\varepsilon_{o}$ is the free-space permittivity and $c$ is the velocity of light. The dispersion characteristic is shown as the full line in Fig. 2(b), for $\omega_{p} / \omega_{t}=0.1$. It applies to an electromagnetic wave incident upon the rod medium with an electric polarization parallel with the direction of the rod. Clearly, the wave is propagating above the cut-off frequency $\omega_{p}$, which may also be referred to as the effective plasma frequency in view of the close analogy to ideal plasmas. Also shown as the dotted line is the characteristic without the rods, which is straight over this range of $k a$.

\section{PERIODIC LOADING OF THE TRANSMISSION LINE BY SRRs}

The split ring resonator may be modeled as a resonator coupled to the electromagnetic wave. In the simplest form, it may be represented as a resonant $L C$ circuit coupled to the transmission line. The coupling is assumed to be purely magnetic. An incident electromagnetic (EM) wave will always excite an emf in the SRR as long as its magnetic field is not in the plane of the rings. For simplicity we shall assume that 

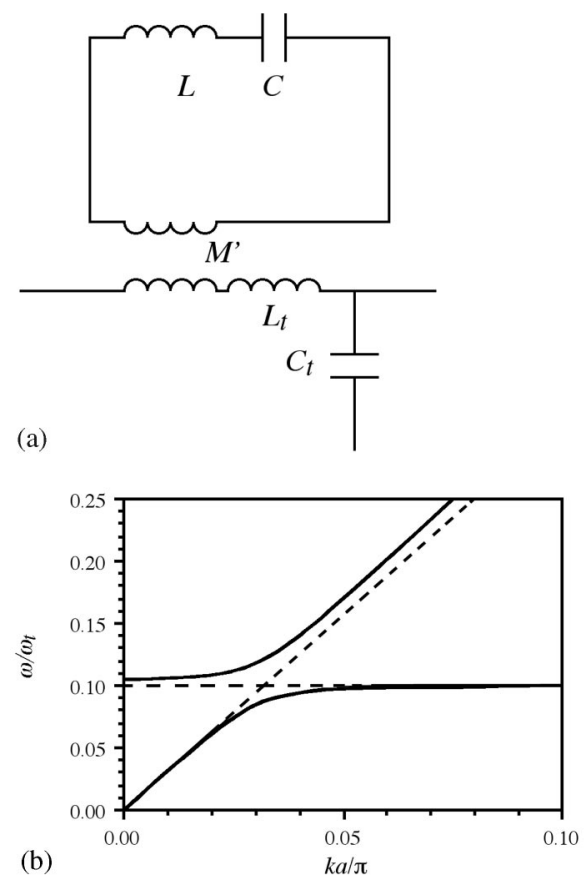

FIG. 3. (a) Equivalent circuit of length $a$ of a transmission line loaded with SRRs; (b) dispersion diagram predicted by Eq. (3) with $\omega_{0} / \omega_{t}=0.1$ and $q^{2}$ $=0$ (dotted line) and (ii) $q^{2}=0.1$ (full line).

the magnetic field is perpendicular to the plane of the rings and that the orientation of the splits is such as to exclude the excitation of an electric dipole moment. A single element of the periodic structure may then be represented by the circuit shown in Fig. 3(a), as proposed by Martin et al. ${ }^{21}$ Here $M^{\prime}$ is a mutual inductance responsible for the coupling, and $L$ and $C$ are the equivalent inductance and capacitance of the SRR. The actual values of $L$ and $C$ were obtained by Marques et $a l^{22}$ by deducing an equivalent circuit from plausible physical assumptions. For a more detailed analysis in terms of distributed and lumped circuit elements, see Refs. 23,24.

Our aim is to assign from the underlying physics a value for every one of our circuit elements. We have so far accounted for $L_{t}, C_{t}, L_{r}, L$, and $C$. The remaining element, $M$ ', will be found (see Appendix) by considering the excitation of the SRR by the magnetic field of the electromagnetic wave.

The dispersion equation corresponding to the periodic circuit shown in Fig. 3(a) can again be obtained by the same method as in the previous section although with a little more effort. We find that,

$$
4 \sin ^{2}(k a / 2)=\left(1-q^{2}\right)\left(\omega^{2} / \omega_{t}^{2}\right)\left[\left(\omega^{2}-\omega_{q}^{2}\right) /\left(\omega^{2}-\omega_{o}^{2}\right)\right] .
$$

Here $q^{2}=M^{, 2} / L L_{t} \ll 1$ is the normalized coupling coefficient, $\omega_{o}{ }^{2}=(L C)^{-1}$, and $\omega_{q}{ }^{2}=\omega_{o}{ }^{2} /\left(1-q^{2}\right)$. This dispersion characteristic is shown as the full lines in Fig. 3(b), for the parameter values $\omega_{o} / \omega_{t}=0.1$ and $q^{2}=0.1$. Also shown as the dotted lines is the equivalent characteristic obtained with $q^{2}=0$, when the transmission line is uncoupled from the SRRs. In this case, there are two curves, one for the EM wave and the other for the SRRs. These curves always cross. When $q^{2}$ $\neq 0$, a stop band is opened near the intersection. As a result, two passbands are created from $\omega=0$ to $\omega=\omega_{o}$ and from $\omega$

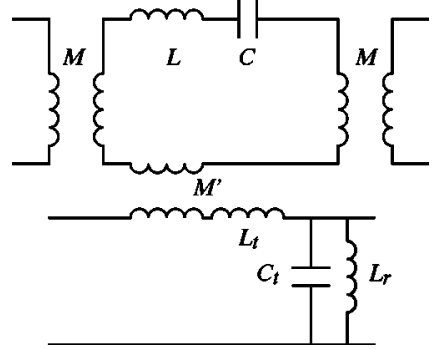

FIG. 4. Equivalent circuit for a transmission line loaded with metal rods and SRRs.

$=\omega_{q}$ upwards, with forward waves propagating in both bands. The stop band ranges from $\omega=\omega_{o}$ to $\omega=\omega_{q}$. Its relative width is equal to

$$
\Delta \omega / \omega_{o}=\left(\omega_{q}-\omega_{o}\right) / \omega_{o}=q^{2} / 2 .
$$

Thus, the fractional band gap is proportional to the normalized coupling coefficient.

\section{COMBINED LOADING BY METALLIC RODS AND SRRS TOGETHER}

\section{A. The general dispersion equation}

We shall now, in Fig. 4, combine the loads shown in Figs. 2(a) and 3(a) and, in addition, we shall take into account that neighboring SRRs are coupled to each other. The magnetic flux generated by one SRR intersects the plane of neighboring SRRs and will consequently induce a voltage in them. This effect can be represented by a mutual inductance $M$ between neighboring elements. If both the size of the element and the period are small relative to the wavelength then the calculation of the mutual inductance between two loops is relatively easy, it can be found in most text books on electromagnetic theory (see, e.g., Ref. 25). The derivation of the dispersion equation for the circuit of Fig. 4 is straightforward again but needs a fair amount of algebraic manipulations. We obtain it in the form of a quadratic equation in $\omega^{2}$,

$$
\begin{aligned}
\omega^{4}[1 & \left.-q^{2}+\kappa \cos (k a)\right]-\omega^{2}\{[1+\kappa \cos (k a)] \\
& \left.\times\left[4 \omega_{t}{ }^{2} \sin ^{2}(k a / 2)+\omega_{p}{ }^{2}\right]+\left(\omega_{o}{ }^{2}-q^{2} \omega_{p}{ }^{2}\right)\right\} \\
& +\omega_{o}{ }^{2}\left[4 \omega_{t}{ }^{2} \sin ^{2}(k a / 2)+\omega_{p}{ }^{2}\right]=0 .
\end{aligned}
$$

Here $\kappa=2 M / L$ is the nearest-neighbor coupling coefficient for the MI waves. We illustrate the use of Eq. (5) with a number of examples.

\section{B. Neighboring SRRs uncoupled, $\kappa=0$}

In this case, the dispersion Eq. (5) may be reduced to the form

$$
\begin{aligned}
4 \sin ^{2}(k a / 2)= & \left(1-q^{2}\right)\left[\left(\omega^{2}-\omega_{p}{ }^{2}\right) / \omega_{t}^{2}\right]\left[\left(\omega^{2}-\omega_{q}{ }^{2}\right) /\right. \\
& \left.\left(\omega^{2}-\omega_{o}{ }^{2}\right)\right] .
\end{aligned}
$$

Equation (6) is clearly very similar to Eq. (3), except that $\omega^{2}$ is replaced by $\omega^{2}-\omega_{p}{ }^{2}$. Note that the same form was obtained (apart from the constants, and in the approximation when $k a \ll 1$ ) by Smith et al., ${ }^{2}$ starting with the expression for the effective value of the permeability ${ }^{5}$ and multiplying it 

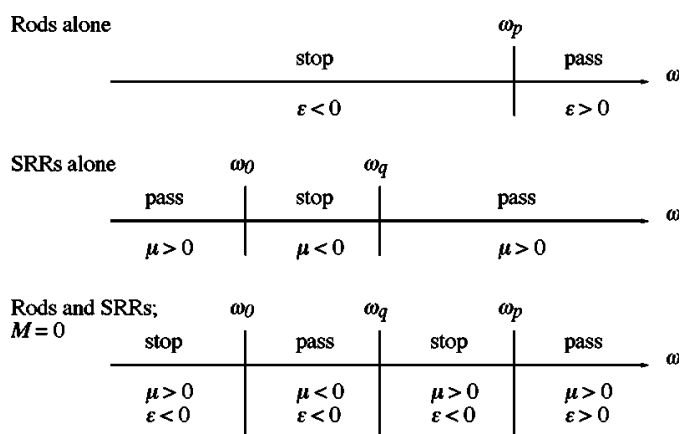

FIG. 5. Representation in frequency space of passband and stop band for loaded transmission lines.

by the effective permittivity of an ideal plasma. It is now clear that ours is an alternative derivation using different physical principles but leading to the same functional relationship.

We shall now summarize in Fig. 5 the different interpretations of the two approaches. The three cases that we have been investigating are represented by three frequency scales, upon which the values of $\omega_{o}, \omega_{q}$, and $\omega_{p}$ are marked. Representations in terms of stop bands and passbands are above the lines and those in terms of effective material parameters (whether they are positive or negative) are below the lines. It may be seen that if the bands generated by each form of load, presented in isolation, are of different kind (i.e., one is a passband and the other one is a stop band), the result for the combined load is a stop band. Conversely, if the bands are of the same kind the result is a passband. In terms of negative material parameters this means, as has been stated many times before in the literature, that there is propagation, provided that both material constants are of the same sign. It also follows from Eq. (6) that when both the SRR and the rods present stop bands on their own, the net effect appears as a passband in which backward waves propagate.

Clearly, Fig. 5 explains the experimental results (i)-(iii) mentioned in the Introduction. Since our analysis is a onedimensional one, negative refraction does not directly follow from it. But it does follow indirectly. We have shown the presence of a backward wave, and Lindell et al. ${ }^{10}$ proved that negative refraction is a consequence of a backward wave incident upon a forward wave medium.

\section{Neighboring SRRs coupled}

Returning now to the general case it may be shown that Eq. (5) may be rewritten in a physically more meaningful form

$$
\begin{aligned}
& \left\{\omega^{2}[1+\kappa \cos (k a)]-\omega_{0}^{2}\right\}\left\{\omega^{2}-\left[\omega_{p}^{2}+4 \omega_{t}^{2} \sin ^{2}(k a / 2)\right]\right\} \\
& \quad=q^{2} \omega^{2}\left(\omega^{2}-\omega_{p}{ }^{2}\right) .
\end{aligned}
$$

When $q^{2}=0$ the left-hand side of Eq. (7) yields two waves propagating independently of each other, one is the electromagnetic wave affected by the rods and the other one is the MI wave. The dispersion equation of the former is given by Eq. (2) whereas that of the latter one is of the form

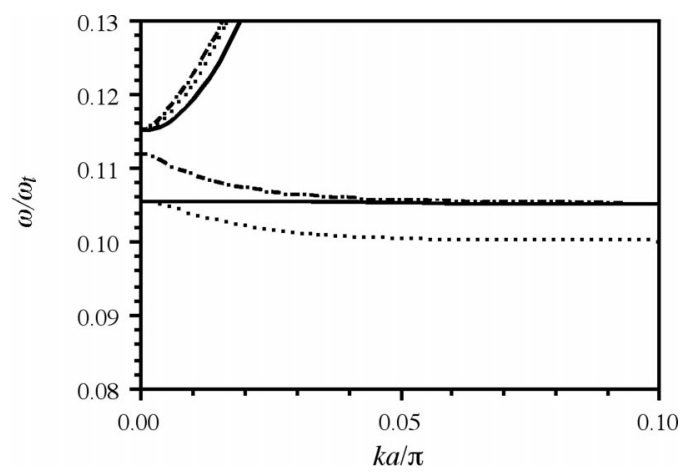

FIG. 6. Dispersion diagrams predicted by Eq. (5) with $\omega_{p} / \omega_{0}=1.15$, $\omega_{0} / \omega_{t}=0.1$ and (i) $\kappa=-0.1$ and $q^{2}=0$ (full line), (ii) $\kappa=0$ and $q^{2}=0.1$ (dotted line), and (iii) $\kappa=-0.1 q^{2}=0.1$ (dot-dash line).

$$
\omega / \omega_{o}=[1+\kappa \cos (k a)]^{-1} .
$$

When $\kappa$ is negative this is a backward wave, as may be seen in Fig. 6 where the dispersion curves are plotted by full lines for $\omega_{0} / \omega_{t}=0.1, \omega_{p} / \omega_{0}=1.15$, and $\kappa=-0.1$. It needs to be emphasized that this is not the backward wave that comes about when both material constants are negative, as discussed in Sec. IV B. That wave may be obtained by taking $\kappa=0$, the same values of $\omega_{p}$ and $\omega_{t}$, and a finite value of $q^{2}$, say, 0.1. The corresponding dispersion curves are plotted in Fig. 6 with dotted lines. So we now have two backward waves, one under the condition that $\kappa=0$ and the other one when $q^{2}=0$. Under experimental conditions we do not normally have the option of choosing one of the coupling constants zero and the other one as finite, they must both be different from zero. To see their joint effect we shall now take their values as $\kappa=-0.1$ and $q^{2}=0.1$, and with $\omega_{p}$ and $\omega_{t}$ remaining the same we plot the resulting dispersion diagrams in Fig. 6 with dot-dash lines. The edge of the backward wave region may be seen to have moved up from 0.105 to 0.112 . We may therefore conclude from Fig. 6 that one of the consequences of the inductive coupling between the elements is a shift in the position of the backward wave region.

Another, equally important, effect is that the EM and MI waves may actually couple to each other. This may become clearer if we choose $\omega_{p}$ so that the dispersion characteristics of EM and MI waves intersect each other when $q^{2}=0$. This is shown in Fig. 7 for $\omega_{0} / \omega_{t}=0.1, \omega_{p} / \omega_{0}=0.9$, and $\kappa=-0.1$

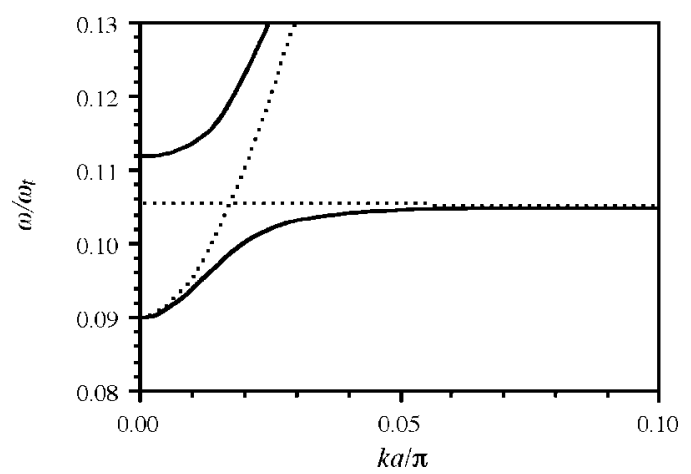

FIG. 7. Dispersion diagrams predicted by Eq. (5) with $\omega_{p} / \omega_{0}=0.9, \omega_{0} / \omega_{t}$ $=0.1, \kappa=-0.1$ and (i) $q^{2}=0$ (dotted line), and (ii) $q^{2}=0.1$ (full line). 
with $q^{2}=0$ for the dotted lines and $q^{2}=0.1$ for the full lines. As may be expected, the interaction leads to the appearance of a stop band.

It would, of course, be possible to investigate many other configurations in which EM and MI waves interact, e.g., when the elements line up at an angle to each other in which case the forward MI wave can be excited. The presence or absence of rods would also make a great difference. The most interesting question to explore is the possibility of exciting a MI wave at one end of the array and gradually transfer its power to an EM wave, a mechanism which may make the launching of an electromagnetic way possible. We intend to return to all these questions in a subsequent paper.

\section{DISCUSSION}

The periodic loading of transmission lines by lumped elements has been successfully applied to the solution of many electromagnetic problems in the last half century. However, with the advent of negative parameter materials a new direction appeared. It was argued by Eleftheriades $e t$ $a l .{ }^{26}$ that the traditional way of representing transmission lines using shunt capacitance and series inductance, as in the present paper, should be reversed in the case of negative parameter materials. In this case, a transmission line should be represented using series capacitance and shunt inductance.

Starting with this principle, Eleftheriades et al. realized that a large number of different circuits may exist which will all show negative refraction, and this claim was demonstrated experimentally by a two-dimensional (2D) realization of loaded transmission lines. Among other things, they demonstrated focusing by these circuit analogs. Similar arguments have been used by Alu and Engheta, ${ }^{27}$ who modeled a negative-permeability material by a negative inductance, and a negative-permittivity material by a negative capacitance. Using these concepts, they have offered explanations of resonances and tunneling in adjacent materials which can have various combinations (both positive and negative) of permittivities and permeabilities.

The major difference between the approaches above and ours is that our circuit models are entirely conventional, so that we can ignore a host of problems that have recently arisen. These include how to treat negative parameter materials, which of the square roots to take in the expressions for velocity and for the refractive index, and whether we should worry about causality.

We may now ask, is it a good thing that after several decades Veselago's ideas ${ }^{9}$ of negative refraction, focusing by a slab, inverted Doppler effect, etc., suddenly became subjects of intense investigations? It is all to the good because the idea stimulated further research and a number of phenomena and potential devices have appeared as a consequence. It would, however, be desirable to answer the question whether negative parameter materials have any deeper meaning, i.e., are there any physical phenomena, which can only be explained by negative material parameters or is it simply a useful artifice that often helps physical intuition? We believed the latter to be true, and are prepared to go even further and offer the following conjecture: any experimental results explained by negative material parameters can also be explained without any recourse to them. The crucial point is to look at the properties of backward waves. Engineers, due to their familiarity with devices based on backward waves, regard them as just one of the manifestations of the multifarious nature of electromagnetic phenomena. Physicists are just warming to the idea.

\section{CONCLUSIONS}

Dispersion equations based on the standard method of periodically loaded transmission lines have been derived for a metamaterial structure consisting of metallic rods and split ring resonators. The derivation is simple and does not require the introduction of concepts such as negative permittivity or permeability. It has also been possible to formulate the interaction between electromagnetic and magnetoinductive waves; this may lead to a way of launching electromagnetic waves. The role of negative material parameters has been discussed, and compared with descriptions in terms of backward waves.

\section{APPENDIX: MUTUAL INDUCTANCE $M$ ' AND THE WIDTH OF THE SRR STOP BAND}

The voltage induced in the resonant SRR may be expressed as $j \omega M^{\prime} I_{t}$, where $I_{t}$ is the current flowing in the transmission line. The same voltage can be expressed by field quantities as $j \omega \pi r_{o}{ }^{2} \mu_{o} H_{t}$, where $H_{t}$ is the magnetic field of the electromagnetic wave and $r_{o}$ is the equivalent radius of the SRR. Considering that for the unit cell $H_{t}=I_{t} / a$ we find,

$$
M^{\prime}=\pi r_{o}^{2} \mu_{o} / a \text {. }
$$

The width of the SRR stop band follows then from Eq. (4) as

$$
\Delta \omega / \omega_{o}=q^{2} / 2=M^{, 2} / 2 L L_{t}=\pi^{2} r_{o}{ }^{4} \mu_{o} / 2 L a^{3} .
$$

This is of course the width of the negative permeability region for which both numerical and analytical solutions exist. It is interesting to note that the analytical solutions of Gorkunov et al., ${ }^{28}$ for no interaction between the elements, have exactly the same expression as Eq. (A2). Such agreement is quite remarkable considering that the two approaches are entirely different. That of Gorkunov et al. is based on the concept of polarization and on averaging magnetic fields, neither of which is considered by us.

${ }^{1}$ D. R. Smith, W. J. Padilla, D. C. Vier, S. C. Nemat-Nasser, and S. Schultz, Phys. Rev. Lett. 84, 4184 (2000).

${ }^{2}$ D. R. Smith, D. C. Vier, N. Kroll, and S. Schultz, Appl. Phys. Lett. 77, 2246 (2000)

${ }^{3}$ R. A. Shelby, D. R. Smith, and S. Schultz, Science 292, 77 (2001).

${ }^{4}$ R. A. Shelby, D. R. Smith, S. C. Nemat-Nasser, and S. Schultz, Appl. Phys. Lett. 78, 489 (2001).

${ }^{5}$ J. B. Pendry, A. J. Holden, D. J. Robbins, and W. J. Stewart, IEEE Trans. Microwave Theory Tech. MTT47, 2075 (1999).

${ }^{6}$ W. Rotman, IRE Trans. Antennas Propag. AP10, 82 (1962).

${ }^{7}$ E. Houck, J. B. Brock, and I. L. Chuang, Phys. Rev. Lett. 90, 137401 (2003).

${ }^{8}$ E. Ozbay, K. Aydin, E. Cubukcu, and M. Bayindir, IEEE Trans. Antennas Propag. AP51, 2592 (2003).

${ }^{9}$ V. S. Veselago, Sov. Phys. Usp. 92, 517 (1967).

${ }^{10}$ V. Lindell, S. A. Tretyakov, K. J. Nikoskinen, and S. Ilvonen, Microwave Opt. Technol. Lett. 31, 129 (2001).

${ }^{11}$ R. G. E. Hutter, Beam and Wave Electronics in Microwave Tubes (Van 
Nostrand, Princeton, NJ, 1964).

${ }^{12}$ P. E. Mayes, G. A. Deschamps, and W. T. Patton, Proc. IRE, 49, 962 (1961).

${ }^{13}$ L. Brillouin, Wave Propagation in Periodic Structures (McGraw-Hill, London, 1946).

${ }^{14}$ R. E. Collin, Field Theory of Guided Waves (Oxford University Press, Oxford, 1991).

${ }^{15}$ M. C. K. Wiltshire, E. Shamonina, I. R. Young, and L. Solymar, Electron. Lett. 39, 215 (2003).

${ }^{16}$ M. C. K. Wiltshire, E. Shamonina, I. R. Young, and L. Solymar, J. Appl. Phys. 95, 4488 (2004).

${ }^{17} \mathrm{M}$. Shamonin and D. J. Edwards (private communications).

${ }^{18}$ E. Shamonina, V. A. Kalinin, K. H. Ringhofer, and L. Solymar, Electron. Lett. 38, 371 (2002).

${ }^{19}$ E. Shamonina, V. A. Kalinin, K. H. Ringhofer, and L. Solymar, J. Appl. Phys. 92, 6252 (2002).

${ }^{20} \mathrm{~F}$. W. Grover, Inductance Calculations: Working Formulas and Tables
(Instrument Society of America, Research Triangle Park, NC, 1981).

${ }^{21}$ R. Martin, J. Bonache, F. Falcone, M. Sorolla, and R. Marques, Appl. Phys. Lett. 83, 4652 (2003).

${ }^{22}$ R. Marques, F. Mesa, J. Martel, and F. Medina, IEEE Trans. Antennas Propag. AP51, 2572 (2003).

${ }^{23}$ M. Shamonin, E. Shamonina, V. Kalinin, and L. Solymar, J. Appl. Phys. 95, 3778 (2004)

${ }^{24}$ L. Solymar, E. Shamonina, M. Shamonin, and V. Kalinin, presented at the Progress in Electromagnetics Research Symposium (PIERS), Honolulu, Hawaii, USA 13-16 October 2003 (unpublished).

${ }^{25}$ L. Solymar, Electromagnetic Theory (Oxford University, Oxford, 1974).

${ }^{26} \mathrm{G}$. V. Eleftheriades, A. K. Tyler, and P. C. Kremer, IEEE Trans. Microwave Theory Tech. MTT50, 2702 (2002).

${ }^{27}$ A. Alu and N. Engheta, IEEE Trans. Antennas Propag. AP51, 2558 (2003).

${ }^{28}$ M. Gorkunov, M. Lapine, E. Shamonina, and K. H. Ringhofer, Eur. Phys. J. B 28, 263 (2000). 